\title{
ADESÃO DE HIPERTENSOS E DIABÉTICOS ANALFABETOS AO USO DE MEDICAMENTO A PARTIR DA PRESCRIÇÃO PICTOGRÁFICA
}

\author{
HYPERTENSIVE AND DIABETIC PATIENT DRUG USE COMPLIANCE BASED ON THE \\ PICTOGRAPHIC PRESCRIPTION
}

\section{ADHESIÓN DE HIPERTENSOS Y DIABÉTICOS ANALFABETOS AL USO DE MEDICAMENTO A PARTIR DE LA PRESCRIPCIÓN PICTOGRÁFICA}

\author{
Guilherme Souza Cavalcanti de Albuquerque ${ }^{1}$ \\ Bárbara do Nascimento ${ }^{2}$ \\ Diego Fabian Karvat Gracia ${ }^{3}$ \\ Luisa Preisler ${ }^{4}$ \\ Paulo de Oliveira Perna ${ }^{5}$ \\ Marcelo José de Souza e Silva ${ }^{6}$
}

Resumo A baixa adesão ao tratamento medicamentoso constitui grave entrave para o sucesso do controle de doenças como o diabetes e a hipertensão arterial. Este artigo trata de um estudo que partiu da identificação do analfabetismo como importante causa de não adesão ao tratamento medicamentoso para diabéticos e hipertensos participantes do programa Hiperdia em unidade de saúde do município de Colombo, no estado do Paraná. Teve como objetivo avaliar o impacto de prescrição pictórica na adesão ao tratamento. Analisou-se um grupo de 63 diabéticos e hipertensos que participavam do Hiperdia, para os quais aplicou-se uma entrevista estruturada, com posterior análise do conteúdo, antes e depois da implantação de uma prescrição pictográfica. Como resultado, entre os analfabetos, observou-se a elevação da adesão de $60 \%$ para $93,33 \%$ em relação ao tratamento medicamentoso. No grupo de alfabetizados não houve mudança na adesão. Conclui-se, no entanto, que esse tipo de intervenção é limitado para a melhora da condição de saúde dos pacientes, pois o aumento da adesão se dá em relação tanto ao tratamento medicamentoso quanto à precariedade.

Palavras-chave adesão ao tratamento medicamentoso; Hiperdia; analfabetismo; determinação social da saúde; precariedade.
Abstract The low levels of compliance with drug therapy is a serious obstacle to the successful control of diseases such as diabetes and hypertension. This article addresses a study that was based on the identification of illiteracy as a major cause of non-compliance with a drug treatment among diabetic and hypertensive patients participating in the Hiperdia program at a health unit in the city of Colombo, state of Paraná, Brazil. The goal was to assess the impact of pictorial prescriptions on compliance with treatment. A group of 63 diabetic and hypertensive patients participating in Hiperdia was analyzed. They answered a structured interview, the content of which was subsequently analyzed before and after the implementation of a pictographic prescription. As a result, compliance with the drug treatment among the illiterate rose from 60 percent to 93.33 percent. There was no change in compliance in the literate group. It was concluded, however, that this type of intervention is limited to the improvement of the patients' health condition, because the increase in compliance occurs both with regard to the drug treatment and precariousness.

Keywords Compliance with drug treatment; Hiperdia; illiteracy; social determinants of health; precariousness. 


\section{Introdução}

As mudanças no perfil de morbimortalidade no Brasil não têm ocorrido da mesma forma como nos países pertencentes ao que se chama de Primeiro Mundo. Motivado por mudanças sociais, demográficas e econômicas ocorridas nos últimos anos, ao lado de políticas sociais redistributivas injustas, nosso país, além de não conseguir se livrar do flagelo das doenças infecciosas, experimentou ainda nesse período um avanço significativo das doenças crônico-degenerativas (Schramm et al., 2004; Goulart, 2011).

Dentre essas doenças, o diabetes e a hipertensão arterial (HA) constituem condições mórbidas de alta prevalência e relevância em nosso meio. Estima-se que existam, atualmente no Brasil, cerca de seis milhões de portadores de diabetes e 17 milhões de portadores de hipertensão arterial, com uma tendência de crescimento desses agravos (Brasil, 2013a, 2013b).

Em decorrência desse panorama, instituiu-se, no Sistema Único de Saúde (SUS), uma ação programada de cuidado a essas doenças, denominada Hiperdia. Trata-se de um sistema de cadastramento e acompanhamento de hipertensos e diabéticos que gera informações para o acompanhamento da assistência a essas pessoas, principalmente visando à garantia do recebimento dos medicamentos prescritos. Assim, o sistema busca a melhoria da qualidade de vida dessas pessoas e a redução do custo social, além de redefinir o perfil epidemiológico da população, a fim de desencadear estratégias de saúde pública para a modificação do quadro atual (Brasil, 2013c).

Segundo essa lógica, e tendo em vista que o tratamento do diabetes e da hipertensão arterial visa especialmente ao controle do valor glicêmico e dos níveis de pressão sanguínea, a adesão ao tratamento medicamentoso e às medidas de higiene preconizadas é fundamental para o sucesso do controle dessas doenças (Shea et al., 1992; Dias et al., 2011; Girotto et al., 2013).

Tanto o diabetes quanto a hipertensão arterial apresentam difícil motivação por parte dos pacientes para adesão ao tratamento, em grande medida por constituírem agravos que não apresentam desconforto físico imediato ou risco evidente para o paciente. Ambas as doenças dependem, para seu controle, de mudanças no estilo de vida, tais como restrições alimentares importantes e o uso contínuo de medicamentos, para se obter não a cura, mas tão somente a atenuação ou retardo do aparecimento de complicações.

Em relação ao tratamento medicamentoso, convencer o paciente sobre a aderência a esse tipo de tratamento depende especialmente das características da terapia, das condições singulares do paciente, seu relacionamento com a equipe médica, a qualidade da terapia fornecida pelos profissionais de saúde, assim como as variáveis psicossociais e socioeconômicas (Wagner, Schnoll e Gipson, 1998; Chin et al., 2000; Alves e Calixto, 2012; Matta, 2010). 
De acordo com Alfonso (2004), na literatura sobre esse assunto os termos adesão e aderência são usados com o significado da ação ativa e voluntária do paciente, segundo a proposta terapêutica que objetiva alcançar resultados intencionais, como simples cumprimento de recomendações médicas. A autora considera aderência terapêutica a expressão mais adequada, porque contempla a dimensão volitiva envolvida com a questão e a define como um comportamento complexo que consiste em uma combinação de aspectos comportamentais com outros relacionais e volitivos. Tal análise leva à participação e ao entendimento do tratamento pelo paciente assim como do plano para sua realização, de maneira conjunta com o profissional de saúde e a consequente resposta obtida por uma busca ativa e consciente de recursos para se alcançar o resultado esperado. A autora esclarece também que o uso dos medicamentos conforme a prescrição médica, por parte daqueles pacientes que estejam mobilizados para tal, depende, entre outras coisas, da compreensão e fixação do modo de uso deles. Tais pressupostos envolvem, nas relações estabelecidas pelos profissionais de saúde com os pacientes, a leitura, a compreensão e o seguimento da receita médica.

Durante as atividades realizadas por estudantes de medicina da Universidade Federal do Paraná (UFPR), participantes do projeto de extensão “Integração ensino/serviço/comunidade no SUS" e do "Programa de Educação pelo Trabalho (PET)-Saúde", em unidade de saúde da família (USF) do SUS no município de Colombo, no estado do Paraná, observou-se baixa adesão ao tratamento medicamentoso dos pacientes do programa Hiperdia, em especial dos analfabetos.

A cidade de Colombo, que integra a região metropolitana de Curitiba, possui 208.805 habitantes, de acordo com o censo de 2010 do Instituto Brasileiro de Geografia e Estatística (Brasil, 2010). Segundo o Instituto, 7,2\% da população com idade superior a 15 anos é analfabeta (Chin et al., 2000; Alves e Calixto, 2012). Embora este índice esteja abaixo da média brasileira, que é de 9,7\% (Brasil, 2010), é bastante superior ao da região sul do país $(5,5 \%)$ e ao da capital paranaense $(3,4 \%)$. Mesmo áreas de maior precariedade quanto às condições de vida na cidade de Curitiba apresentam índices em torno dos $6 \%$ de analfabetos, conforme estudo da Terra de Direitos, organização que atua na defesa e promoção de direitos humanos (Oliveira, Barreto e Litka, 2011).

Com base nessa constatação e de modo a interferir positivamente no tratamento medicamentoso dos pacientes, instituiu-se uma prescrição própria para analfabetos, visando melhorar a adesão a esse tipo de tratamento daqueles pacientes incluídos no programa Hiperdia. Assim, o presente trabalho, desenvolvido no Núcleo de Estudos de Saúde Coletiva da Universidade Federal do Paraná (Nesc-UFPR), teve por objetivo avaliar o impacto de 
tal medida na adesão ao tratamento medicamentoso dos pacientes analfabetos participantes do Hiperdia de uma unidade de saúde do município de Colombo.

\section{Percurso metodológico: do concreto caótico ao concreto pensado}

O fundamento metodológico utilizado foi o do materialismo histórico-dialético, com o intuito de analisar a realidade além de sua aparência, de modo a compreender os fenômenos observados como expressão particular de uma generalidade que os explica e os determina.

Desenvolveu-se, nesse sentido, um esforço para estruturar a pesquisa de modo a superar a observação da realidade como um concreto 'caótico'. Por meio de abstrações, buscou-se compreendê-la como síntese de múltiplas determinações, constituintes do modo de produção (capitalista) no qual estão inseridas, para se perceber a pertinência das propostas de solução apresentadas.

Inicialmente realizou-se um estudo descritivo qualitativo, no qual os dados foram obtidos após o consentimento dos 63 pacientes diabéticos e hipertensos que participavam do Hiperdia, durante uma das reuniões. Aplicou-se uma entrevista estruturada, na qual se indagava a forma de utilização dos medicamentos, diante da prescrição médica em mãos. Os pacientes foram entrevistados individualmente, em consultório, após aferição da pressão arterial (PA) e entrega dos medicamentos. Posteriormente procedeu-se à análise de conteúdo, verificando-se a adesão ao tratamento medicamentoso entre os alfabetizados e os analfabetos.

Foram considerados alfabetizados aqueles pacientes capazes de ler a receita. Aqueles que não conseguiram ler a prescrição apontaram a sua condição de analfabetos como principal fator para a não adesão ao tratamento medicamentoso.

Discutiram-se então, com os pacientes, possíveis formas de melhorar a compreensão da prescrição. Optou-se por uma prescrição médica baseada em pictogramas (Matos, 2009; Medeiros et al., 2011) e cores, na qual cada pictograma representava um período do dia (manhã, tarde ou noite), e círculos com cores distintas indicavam cada medicamento. As cartelas de medicamentos foram marcadas com fita colorida. Na prescrição, assinalava-se determinado número de círculos com a coloração correspondente à fixada em cada cartela.

Dessa maneira, o usuário, ao retirar o medicamento prescrito, previamente marcado com determinada cor, relacionava-o ao período do dia representado pelos pictogramas e ao número de círculos coloridos (correspondentes ao número de comprimidos) marcados em sua prescrição. Deve-se ressaltar que mesmo os pacientes alfabetizados foram orientados quanto ao uso dos 
medicamentos, e alguns deles, com dificuldades para seguir a prescrição, também preferiram a receita baseada em pictogramas.

A avaliação da adesão ocorreu em uma fase posterior, com base nas informações dos pacientes quanto ao modo de uso dos medicamentos e na verificação do número de comprimidos restantes, desde a última entrega. Foi considerado aderente ao tratamento aquele paciente que fazia uso regular da medicação, conforme a prescrição médica. Essa avaliação se deu pela comparação com dados pré-intervenção, coletados nas reuniões realizadas nos meses de maio e junho de 2012. Durante a reunião de junho, e a partir de então, a nova prescrição foi entregue aos participantes analfabetos.

Os primeiros dados pós-intervenção foram obtidos após dois meses, em nova entrevista, durante as novas reuniões e consultas. Os dados mais recentes foram obtidos seis meses depois da implantação da prescrição com os pictogramas.

Após sistematização e análise dos dados coletados, buscou-se dimensionar o impacto da ação para além do âmbito singular imediato. Com suporte no referencial metodológico citado inicialmente, realizou-se uma análise dos resultados com relação à contribuição para a adaptação dos pacientes à sua condição não saudável de vida, ou para a superação daquela condição.

Para a realização da presente pesquisa, a população estudada foi constituída pelos usuários participantes do programa Hiperdia de uma das três áreas de uma USF do SUS do município de Colombo, no estado do Paraná, cumpridos todos os princípios éticos contidos na Declaração de Helsinki (World Medical Association, 2008), da World Medical Association, e da resolução n. 196/96 para as questões éticas na pesquisa.

Este estudo foi aprovado pelo Comitê de Ética da Universidade Federal do Paraná, Setor de Ciências da Saúde, registrado como CEP/SD n. 772.107.09.08, com Certificado de Apresentação para Apreciação Ética (CAAE) n. 0049.0.091.091-09. Ressalta-se que não existem conflitos de interesse associados com esta publicação e não houve qualquer suporte financeiro para este trabalho.

\section{Adesão ao tratamento e à precariedade}

Com base nos dados do programa Hiperdia dos meses de maio e junho de 2012 (pré-intervenção), observou-se que, de um total de 63 hipertensos que estiveram na USF naquele período, trinta eram analfabetos e 33 alfabetizados. Dentre os analfabetos, 18 (60\%) aderiam ao tratamento recomendado, utilizando corretamente a medicação prescrita, enquanto no grupo de alfabetizados, 31 participantes $(93,93 \%)$ eram aderentes ao tratamento medicamentoso. Após observação dos dados referentes a julho e agosto (pós-intervenção), 
verificou-se que, dos trinta pacientes analfabetos inicialmente avaliados, 28 participantes $(93,33 \%)$ tornaram-se aderentes ao tratamento. No grupo dos participantes alfabetizados, não houve mudança quanto à adesão, que continuou correspondendo a 93,93\% dos que se apresentaram no início do trabalho.

Em avaliação realizada no mês de novembro, seis meses após o início da intervenção inicial, os dados revelaram ligeira redução da adesão (87\% entre os alfabetizados e $79 \%$ entre os analfabetos), porém, mesmo assim, ainda bastante superior aos percentuais anteriores ao processo - o que demonstrou a necessidade de processo educativo permanente para os usuários do serviço de saúde.

Souza (2004), em estudo desenvolvido no município de Contenda, no estado do Paraná, e Reiners e colaboradores (2012), em estudo desenvolvido em Cuiabá, no estado do Mato Grosso, relatam que pacientes analfabetos têm grande dificuldade em aderir aos tratamentos propostos. $\mathrm{O}$ primeiro ponto de apoio para a adesão é a família e, em seguida, a equipe de saúde. De acordo com Souza (2004), esses profissionais conhecem a realidade do usuário e consideram as condições reais que os envolvem no momento de traçar as metas para êxito do tratamento. Esforçam-se para aperfeiçoar recursos e estratégias e, assim, manter a qualidade de vida do paciente, atuando conforme as necessidades e capacidades do usuário.

Assim como as baixas taxas de expectativa de vida (Messias, 2003), o analfabetismo está presente como fator dificultador da adesão ao tratamento medicamentoso (Matos, 2009; Medeiros et al., 2011; Marques et al., 2010), pois, por mais que pacientes tenham interesse em modificar hábitos de vida e desejem seguir a prescrição e orientação da equipe de saúde, há momentos em que isso se torna impossível, pela incapacidade de leitura e compreensão da prescrição.

A medicação fornecida na USF é padronizada, mas as embalagens e a coloração dos comprimidos são variáveis, o que dificulta ainda mais a adesão ao tratamento medicamentoso do paciente analfabeto. Muitos deles relataram que, para esse problema, contavam com o auxílio da família e dos próprios profissionais de saúde - que separavam as medicações em sacolas diferentes ou com fitas coloridas para que o paciente não tomasse a medicação incorreta. Matos (2009) ressalta que a adesão por parte de pacientes analfabetos se diferencia da adesão dos alfabetizados, uma vez que a escolaridade dos últimos facilita o uso correto dos medicamentos e a independência do paciente em relação a familiares e profissionais da saúde. Essa associação com o nível de escolaridade também foi encontrada em estudos como o desenvolvido por Gimenes, Zanetti e Haas (2009) em São Paulo, no qual se constatou que a adesão ao tratamento foi maior entre os pacientes com mais de 12 anos de estudo (88,9\%) do que entre os que possuíam me-nos de 12 anos de estudo, cujo percentual de adesão encontrado foi de 75,7\%. 
Dessa forma, assim como em nosso estudo, os resultados daquele sugerem que ações que favoreçam a compreensão da prescrição, sem a necessidade da leitura, possivelmente contribuiriam para maior adesão ao tratamento.

Observou-se, com base nos resultados apresentados, que em nossa pesquisa a adoção da prescrição modificada, com o apoio de pictogramas e círculos coloridos, auxiliou os pacientes analfabetos no uso correto da medicação prescrita para tratamento da hipertensão arterial ou diabetes. A adesão do grupo de pacientes que participou do estudo passou de $60 \%$ para 93,33\% após a implantação da prescrição modificada, mantendo-se em torno de $80 \%$ após seis meses de uso da nova forma de prescrição. O pequeno tamanho da população estudada, no entanto, permitiu apenas que apontássemos uma forte tendência entre a intervenção realizada e o desfecho encontrado, não uma relação causal evidente.

A adesão é fundamental no tratamento medicamentoso de doenças crônicas como a hipertensão arterial e o diabetes, visando ao controle e à prevenção ou retardo no surgimento de complicações. No entanto, a determinação da adesão de pacientes ao tratamento medicamentoso precisa ser analisada nos âmbitos geral, particular e singular, considerando que sua compreensão está necessariamente associada ao estudo sistemático dos mesmos processos que, segundo Breilh (1991), devem presidir o estudo do processo saúde-doença: os processos estruturais da sociedade, que constituem o âmbito geral; os perfis de reprodução social (produção e consumo) das diferentes classes e frações de classes, que constituem o âmbito particular; e a compreensão integral de fenômenos biológicos e comportamentais que compõem os padrões típicos de saúde desses grupos e de seus indivíduos, que constituem o âmbito singular.

Nessa perspectiva, observa-se que a intervenção realizada nos pacientes do Hiperdia ficou restrita ao âmbito singular, restrita ao comportamento dos indivíduos. A alternativa encontrada para a compreensão da prescrição por meio dos símbolos - que, para o analfabeto, aumenta indubitavelmente a possibilidade de adesão ao uso do medicamento - deixa intocadas, no entanto, duas questões cruciais: não contribui para retirar os pacientes da condição de analfabetismo, condição gerada pela expropriação dessa parcela da população de importante objeto humano de comunicação que é a linguagem escrita; e não contribui diretamente em nada para modificar a condição de precariedade daquela população, privada de muitos dos objetos produzidos pela humanidade, para além do domínio da leitura. Trata-se de pessoas com carências relacionadas a questões muito elementares, imprescindíveis para a realização da vida com dignidade, como a posse da terra ou da casa própria, o emprego, o salário suficiente, o saneamento ambiental, o controle de vetores, o acesso à escola, áreas de lazer, serviços de saúde e segurança, meios de transporte e comunicação. 
Como afirma Klein (2011):

Nesse contexto de negação generalizada dos direitos, torna-se evidente a dificuldade de realização do direito à educação, posto que (...) tal direito exige um contexto de amplo usufruto de práticas sociais, as mais ricas e diversificadas, donde a sua efetivação depende de um complexo de recursos materiais concretos, expropriados dos trabalhadores, que só podem por eles serem acessados pela via da intervenção estatal (Klein, 2011, p. 11-12).

Em última instância e contraditoriamente, a ação favorável à manutenção da vida contribui, ao mesmo tempo, para a adaptação daqueles sujeitos a uma condição de precariedade, a um modo de vida marcado pela privação dos bens e serviços essenciais. Auxilia a mantê-los vivos, mas não a conquistar melhores condições gerais de existência.

Entende-se que é fundamental investir todo esforço para manter a vida, mas isso não é tudo. Ao profissional da saúde cabe, necessariamente, procurar oferecer aos indivíduos condições de plena realização de suas potencialidades.

As ações realizadas no âmbito da saúde pública, mesmo aquelas orientadas pelos princípios da saúde da família, costumam restringir seu campo de intervenção ao domínio individual (incluídas aí a estrutura e a dinâmica familiar do indivíduo). Apesar da contribuição trazida por esse tipo de intervenção, para se obterem resultados consistentes, é preciso superar os limites do singular com o enfrentamento das questões macro, determinantes do processo saúde-doença (Buss, 2009).

A despeito das boas intenções, as ações de saúde pública, por constituírem ações de Estado, não conseguem ultrapassar os limites da reprodução da ordem. Carecem de reflexões sobre a desigualdade social produzida pelo capitalismo, em que as estratégias de inclusão ocorrem de forma precarizada, guiadas apenas pelo interesse econômico de reprodução da força de trabalho (Jorge, 2011).

Nesse sentido, o ideário da 'promoção da saúde' tem procurado avançar. Tal ideia surge como reação à grande medicalização, tanto na sociedade em geral quanto no interior do sistema sanitário, pretendendo dar um enfoque político e técnico sobre o processo saúde-doença-cuidado (Buss, 2009).

Segundo Buss (2009) e Czeresnia (2009), o citado ideário, em uma abordagem moderna, mais atual, compreende o protagonismo dos determinantes gerais em relação aos determinantes individuais sobre as condições de saúde, tendo como objetivo que os indivíduos e os coletivos alcancem condições de vida dignas e adequadas, com um nível de vida ótimo, não sendo suficiente apenas a ausência de doenças.

Dessa forma, Buss (2009) define a promoção da saúde segundo uma concepção ampliada: 
A saúde é produto de um amplo espectro de fatores relacionados com a qualidade de vida, incluindo um padrão adequado de alimentação e nutrição, de habitação e saneamento, boas condições de trabalho, oportunidades de educação ao longo de toda a vida, ambiente físico limpo, apoio social para famílias e indivíduos, estilo de vida responsável e um espectro adequado de cuidados de saúde (Buss, 2009, p. 23).

Para o referido autor, a promoção da saúde está, portanto, voltada mais para o coletivo de indivíduos e o ambiente, a serem contemplados por políticas públicas saudáveis. Para tal, faz-se necessária a capacitação da comunidade, visando a uma atuação mais consistente na melhoria da sua própria qualidade de vida.

As políticas públicas para a promoção da saúde realizam-se por meio de diversas abordagens distintas, como "legislação, medidas fiscais, taxações e mudanças organizacionais, entre outras, e por ações coordenadas que apontam para a equidade em saúde, distribuição mais equitativa da renda e políticas sociais" (Buss, 2009, p. 31). As políticas para ambientes que promovam a saúde devem ser aquelas que favoreçam vidas saudáveis para as pessoas e que também facilitem opções saudáveis de vida, incluindo nessa área não apenas a dimensão física de ambiente, mas também as dimensões social, econômica, política e cultural.

No caso aqui apresentado, não houve intervenção nos âmbitos particular e geral. A intervenção nesses níveis foge da governabilidade da ação tradicional tanto do médico quanto dos outros profissionais de saúde, ou até mesmo dos próprios serviços de saúde voltados para os indivíduos.

Para provocar mudanças na instância particular, as ações teriam que estar voltadas para as condições de existência do grupo de usuários analfabetos do programa Hiperdia, da USF estudada.

Não se trata de medicalizar a questão. Não se trata de depositar sobre a saúde a responsabilidade pela solução dos problemas que a sociedade como um todo produz - fato que, contraditoriamente, é o que mais usualmente ocorre, mesmo quando se parte do pressuposto de que as 'questões sociais' não são de responsabilidade da saúde. A pretensão de buscar solucionar a hipertensão, assim como a drogadição, a gravidez na adolescência, entre outras condições, com as armas do atendimento médico, clínico, individual, ou mesmo um cuidado realizado por equipe multiprofissional, segundo a lógica hegemônica dos serviços de saúde, é que constitui uma ação de medicalização (Illich, 1975).

Segundo Oliveira, Barreto e Litka (2011):

A marginalização dos jovens e adultos que não possuem estudo formal concluído em idade adequada está explícita nas relações sociais determinadas pelo modo de 
produção atual. No espaço destinado a eles no mercado de trabalho, no acesso à cultura, tecnologia, lazer, seletivamente ofertados, é possível perceber a dinâmica social de concentração das riquezas e exclusão dos sujeitos (Oliveira, Barreto e Litka, 2011, p. 1).

Uma contribuição significativa para a saúde, entendida como condição potencial de plena realização do ser humano (Albuquerque e Silva, 2014), exige superar os limites estreitos da intervenção nos indivíduos e da responsabilização deles por sua condição de saúde; exige demonstrar os nexos de determinação entre o singular, o particular e o geral. Também exige ações de prevenção profunda da saúde (Breilh, 2006). Explicitar que cada indivíduo realiza sua existência nos limites impostos por sua condição particular de classe ou fração de classe - condição esta determinada, também, pelos limites impostos pela nossa ordem social capitalista - contribuiria significativamente para o movimento necessário de superação desse modo de produção patogênico e limitador da plena realização do ser humano.

\section{Considerações finais}

A ação tradicional dos serviços de saúde realiza a medicalização da sociedade, transfigurando problemas de origem social em problemas médicos. A obrigação de resolvê-los é transferida aos serviços e profissionais da saúde, ocultando sua determinação social.

Neste trabalho, a intervenção adotada em relação aos analfabetos localizava-se nos limites de uma política pública de Estado (a saúde pública), instituindo ações pertinentes à instância singular, necessárias, mas insuficientes, pois carecem da discussão crítica e da denúncia da existência de gente privada de coisas indispensáveis para uma existência humana civilizada, gente precarizada.

Com essa reflexão, no entanto, não negamos que a prescrição adaptada, por melhorar de forma considerável a adesão dos pacientes analfabetos ao medicamento, apresenta-se como um importante recurso para o tratamento de hipertensão arterial e diabetes no citado grupo. Para lutar pela transformação da sociedade, é preciso, antes de tudo, estar vivo. 


\section{Colaboradores}

Guilherme Souza Cavalcanti de Albuquerque participou do delineamento, análise e interpretação dos dados, redação do artigo e revisão crítica; Bárbara do Nascimento, da concepção, delineamento, análise e interpretação dos dados, e redação do artigo; Diego Fabian Karvat Gracia participou da concepção, delineamento, análise e interpretação dos dados, e redação do artigo; Luisa Preisler, da redação do artigo e revisão crítica; Paulo de Oliveira Perna participou da revisão crítica do artigo; Marcelo José de Souza e Silva, da redação do artigo e revisão crítica. Todos os autores aprovaram a versão a ser publicada.

Resumen La baja adhesión al tratamiento medicamentoso constituye un grave obstáculo para el éxito del control de enfermedades como la diabetes y la hipertensión arterial. Este artículo trata de un estudio que partió de la identificación del analfabetismo como importante causa de la no adhesión al tratamiento medicamentoso para diabéticos e hipertensos participantes en el programa Hiperdia en una unidad de salud del municipio de Colombo, estado de Paraná, Brasil. Éste tuvo como objetivo evaluar el impacto de la prescripción pictórica en la adhesión al tratamiento. Se analizó un grupo de 63 diabéticos e hipertensos que participaban en el Hiperdia, para los cuales se aplicó una entrevista estructurada, con posterior análisis del contenido, antes y después de la implantación de una prescripción pictográfica. Como resultado, entre los analfabetos, se observó la elevación de la adhesión del 60\% para el 93,33\% con relación al tratamiento medicamentoso. En el grupo de alfabetizados, la adhesión no presentó cambios. Se concluyó, sin embargo, que este tipo de intervención es limitado para la mejora de la condición de salud de los pacientes, pues el aumento de la adhesión se da con relación al tratamiento medicamentosos en cuanto a la precariedad. Palabras clave adhesión al tratamiento medicamentoso; Hiperdia; analfabetismo; determinación social de la salud; precariedad.

\section{Notas}

1 Universidade Federal do Paraná, Departamento de Saúde Comunitária, Núcleo de Estudos em Saúde Coletiva, Curitiba, Paraná, Brasil.

<guilherme.albuquerque@ufpr.br>

Correspondência: Universidade Federal do Paraná, Departamento de Saúde Comunitária, Núcleo de Estudos em Saúde Coletiva, Rua Padre Camargo, 280, $7^{\circ}$ andar, CEP 80060-240, Alto da Glória, Curitiba, Paraná, Brasil. 
2 Universidade Federal do Paraná, Departamento de Saúde Comunitária, Núcleo de Estudos em Saúde Coletiva, Curitiba, Paraná, Brasil.

$<$ b_nascimento@hotmail.com>

3 Universidade Federal do Paraná, Departamento de Saúde Comunitária, Núcleo de Estudos em Saúde Coletiva, Curitiba, Paraná, Brasil.

<topdiego@ig.com.br>

4 Universidade Federal do Paraná, Departamento de Saúde Comunitária, Núcleo de Estudos em Saúde Coletiva, Curitiba, Paraná, Brasil.

$<$ luisapreisler@gmail.com>

5 Universidade Federal do Paraná, Departamento de Saúde Comunitária, Núcleo de Estudos em Saúde Coletiva, Curitiba, Paraná, Brasil.

<perna@ufpr.br>

6 Universidade Federal do Paraná, Departamento de Saúde Comunitária, Núcleo de Estudos em Saúde Coletiva, Curitiba, Paraná, Brasil.

$<$ marcelojss@gmail.com>

\section{Referências}

ALBUQUERQUE, Guilherme S. C.; SILVA, Marcelo J. S. Sobre a saúde, os determinantes da saúde e a determinação social da saúde. Saúde em Debate, Rio de Janeiro, v. 38, n. 103, p. 953-965, 2014.

ALFONSO, Libertad M. Acerca del concepto de adherencia terapéutica. Revista Cubana de Salud Pública, Ciudad de La Habana, v. 30, n. 4, set.-dez. 2004. Disponível em: <http:// scielo.sld.cu/scielo.php?script=sci_arttext\& pid $=$ S0864-34662004000400008 > . Acesso em: 12 jul. 2013.

ALVES, Bruna A.; CALIXTO, Amanda A. T. F. Aspectos determinantes da adesão ao tratamento da hipertensão e diabetes em uma unidade básica de saúde do interior paulista. Journal of the Health Sciences Institute, São Paulo, v. 30, n. 3, p. 255-260, 2012.
BRASIL. Ministério da Saúde. Estratégias para o cuidado da pessoa com doença crônica: diabetes mellitus. Brasília: Ministério da Saúde, 2013a. (Cadernos de Atenção Básica 36).

BRASIL. Ministério da Saúde. Estratégias para o cuidado da pessoa com doença crônica: hipertensão arterial sistêmica. Brasília: Ministério da Saúde, 2013b. (Cadernos de Atenção Básica, 37).

BRASIL. Ministério da Saúde. Hiperdia. 2013. Disponível em: <http://hiperdia.datasus. gov.br>. Acesso em: 12 jul. 2013.

BRASIL. Ministério do Planejamento, Orçamento e Gestão. Instituto Brasileiro de Geografia e Estatística (IBGE). Pesquisa Nacional por Amostra de Domicílios: 2009. Rio de Janeiro: IBGE, 2010. Disponível em: <www. 
ibge.gov.br/home/estatistica/populacao/ trabalhoerendimento/pnad2009/>. Acesso em: 12 jul. 2013.

BREILH, Jaime. Epidemiologia: economia, política e saúde. São Paulo: Unesp; Hucitec, 1991.

BREILH, Jaime. Epidemiologia critica: ciência emancipadora e interculturalidade. Rio de Janeiro: Editora Fiocruz, 2006.

BUSS, Paulo M. Uma introdução ao conceito de promoção da saúde. In: CZERESNIA, Dina; FREITAS, Carlos M. (orgs.). Promoção da saúde: conceitos, reflexões, tendências. 2. ed. rev. e ampl. Rio de Janeiro: Editora Fiocruz, 2009. p. 19-42.

CHIN, Marshall et al. Quality of diabetes care in community health centers. American Journal of Public Health, Washington, DC, v. 90, n. 3, p. 431-434, 2000. Disponível em: <www.ncbi.nlm.nih.gov/pmc/articles/ PMC1446172/pdf/10705866.pdf $>$. Acesso em: 15 jul. 2013.

CZERESNIA, Dina. O conceito de saúde e a diferença entre prevenção e promoção. In: CZERESNIA, Dina; FREITAS, Carlos M. (orgs.). Promoção da saúde: conceitos, reflexões, tendências. 2. ed. rev. e ampl. Rio de Janeiro: Editora Fiocruz, 2009. p. 43-57.

DIAS, Antonio M. et al. Adesão ao regime terapêutico na doença crônica: revisão de literatura. Millenium, Viseu, Portugal, n. 40, p. 201-219, jun. 2011.

GIMENES, Heloisa T.; ZANETTI, Maria L.; HAAS, Vanderlei J. Fatores relacionados à adesão do paciente diabético à terapêutica medicamentosa. Revista Latino-Americana de Enfermagem, Ribeirão Preto, v. 17, n. 1, 2009. Disponível em: <www.scielo.br/pdf/ rlae/v17nl/pt_08.pdf>. Acesso em: 15 jul. 2013.

GIROTTO, Edmarlon et al. Adesão ao tratamento farmacológico e não farmacológico e fatores associados na atenção primária da hipertensão arterial. Ciência \& Saúde Cole- tiva, Rio de Janeiro, v. 18, n. 6, p. 1.7631.772, 2013.

GOULART, Flavio A. A. Doenças crônicas não transmissíveis: estratégias de controle e desafios para os sistemas de saúde. Brasília: Organização Pan-Americana da Saúde; Ministério da Saúde, 2011.

ILLICH, Ivan. A expropriação da saúde: nêmesis da medicina. 3. ed. Rio de Janeiro: Nova Fronteira, 1975.

JORGE, Céuli M. Inclusão social e integração curricular: dilemas e contradições do Proeja. In: ENCONTRO BRASILEIRO DE EDUCAÇÃO E MARXISMO, 5., 2011, Florianópolis. Anais... Florianópolis: Ebem, 2011. Acesso em: 23 jun. 2013.

KLEIN, Bianca L. Escola para o trabalhador: o direito à educação posto à prova. In: ENCONTRO BRASILEIRO DE EDUCAÇÃO E MARXISMO, 5, 2011, Florianópolis. Anais... Florianópolis: Ebem, 2011. Acesso em: 23 jun. 2013.

MARQUES, Elizabeth I. W. et al. Motivos da não adesão ao tratamento médico prescrito entre os idosos de uma unidade de saúde da família do município de Passo Fundo-RS. Revista Brasileira de Ciências do Envelhecimento Humano, Passo Fundo, v. 7, n. 2, p. 267-279, maio-ago. 2010.

MATOS, Ciro R. Pictogramas e seu uso nas instruções médicas: estudo comparativo entre repertórios para instruções de uso de medicamentos. Dissertação (Mestrado em Ciências da Comunicação) - Escola de Comunicações e Artes da Universidade de São Paulo, São Paulo, 2009.

MATTA, Samara R. Adaptação transcultural de instrumento para medida da adesão ao tratamento anti-hipertensivo e antidiabético. Dissertação (Mestrado em Ciências) - Escola Nacional de Saúde Pública Sergio Arouca, Fundação Oswaldo Cruz, Rio de Janeiro, 2010.

MEDEIROS, Giovanna C. R. et al. Pictogramas na orientação farmacêutica: um estudo 
de revisão. Revista Brasileira de Farmácia, Rio de Janeiro, v. 93, n. 2, p. 96-103, 2011.

MESSIAS, Erick. Income inequality, illiteracy rate, and life expectancy in Brazil. American Journal of Public Health, Washington, DC, v. 93, n. 8, p. 1.294-1.296, 2003. Disponível em: <www.ncbi.nlm.nih.gov/pmc/ articles/PMC1447959/pdf/0931294.pdf>. Acesso em: 27 jun. 2013.

OLIVEIRA, Juliana P. F.; BARRETO, Maria L. M. M.; LITKA, Miriany. Tempo livre para o estudo e seu impacto na organização pedagógica em EJA. In: ENCONTRO BRASILEIRO DE EDUCAÇÃO E MARXISMO, 5., 2011, Florianópolis. Anais... Florianópolis: Ebem, 2011. Acesso em: 23 jun. 2013.

REINERS, Annelita A. O. et al. Adesão ao tratamento de hipertensos da atenção básica. Ciência, Cuidado e Saúde, Maringá, v. 11, n. 3, p. 581-587, jul.-set. 2012.

SCHRAMM, Joyce M. A. et al. Transição epidemiológica e o estudo de carga de doença no Brasil. Ciência \& Saúde Coletiva, Rio de Janeiro, v. 9, n. 4, p. 897-908, 2004. Disponível em: <www.scielo.br/pdf/csc/v9n4/ allv9n4.pdf>. Acesso em: 29 jul. 2013.

SHEA, Steven et al. Correlates of nonadherence to hypertension treatment in an inner-city minority population. American Journal of Public Health, Washington, DC, v. 82, n. 12, p. 1.607-1.612, 1992. Disponível em: <www. ncbi.nlm.nih.gov/pmc/articles/PMC1694541/ pdf/amjph00549-0025.pdf>. Acesso em: 27 jun. 2013.
SOUZA, Patrícia M. Hipertensão arterial: analfabetismo e adesão ao tratamento - um estudo realizado com usuários atendidos pelo Programa Saúde da Família na cidade de Contenda, Paraná. 108f. Monografia (Especialização em Saúde Coletiva) - Núcleo de Estudos em Saúde Coletiva (NESC), Setor de Ciências da Saúde, Universidade Federal do Paraná, Curitiba, 2004.

WAGNER, Julie A.; SCHNOLL, Robert; GIPSON, Martin. Development of a scale to measure adherence to self-monitoring of blood glucose with latent variable measurement. Diabetes Care, Alexandria, v. 21, n. 7, p. 1.046-1.051, 1998. Disponível em: < http:// care.diabetesjournals.org/content/21/7/1046. full.pdf>. Acesso em: 28 jun. 2013.

WORLD MEDICAL ASSOCIATION. World Medical Association Declaration of Helsinki: ethical principles for medical research involving human subjects, 2008. Disponível em: $<$ www.wma.net/en/30publications/10policies/ b3/17c.pdf $>$. Acesso em: 13 maio 2013.

Recebido em 12/08/2013

Aprovado em 05/04/2015 